\title{
Bit Error Rate Performance in Power Line Communication Channels with Impulsive Noise
}

\author{
Ali Hosseinpour Najarkolaei \\ University of East London \\ London, UK \\ najarkolaei@ieee.org
}

\author{
Wada Hosny \\ University of East London \\ London, UK \\ w.m.hosny@uel.ac.uk
}

\author{
Jaswinder Lota ${ }^{\perp}$ \\ University of East London $\dagger$ \\ University College London $\perp$ \\ London, UK \\ j.lota@uel.ac.ukt,,j.lota@ucl.ac.uk ${ }^{\perp}$
}

\begin{abstract}
Power Line Communication (PLC) has the potential to become the preferred technique for providing broadband to homes and offices with advantage of eliminating the need for new wiring infrastructure and reducing the cost. However, power line grids present a hostile channel for data communication, since the fundamental purpose for power line channel was only the transmission of electric power at $50 / 60 \mathrm{~Hz}$ frequencies. The performance of PLC degrades due to the presence of different types of noise interferences generated by electrical appliances. This paper investigates the bit-error rate (BER) performance of 16-QAM constellation with orthogonal frequency multiplexing modulation (OFDM) in presence of impulsive noise and background noise over a multipath PLC channel. A Middleton class $A$ noise is modelled as an impulsive noise whereas the background noise is modelled as an Additive White Gaussian Noise (AWGN). Matlab model is developed to access the PLC channel's behavior in presence of these two noises. The effect of varying the Middleton class A model's parameters on the PLC channel's performance is also investigated. It is observed that BER for the impulsive noise is higher than the background noise. The BER further deteriorates on increasing the level of the impulsive noise, even while being injected into the PLC channel at a lower rate. Investigations would assist applying methods to mitigate and reduce the effect of impulsive noise over PLC systems for higher constellations with a view to increase the data rates.
\end{abstract}

Keywords-AWGN; BER; Impulsive Noise; Middleton Class A; PLC; Power Line Communication; OFDM; QAM

\section{INTRODUCTION}

The Power Line Communication (PLC) technology is emerging as an alternative to the broadband access network with the advantage of using the existing infrastructure of power line networks which results a great cost reduction. However the PLC channel is not conducive to high speed data transmission due to attenuation and interferences from various sources [1],[2]. In [3] different types of PLC noise sources are modelled in Matlab/Simulink, among these the background and impulsive noises are the main source of interference resulting in signal distortion. The background noise can be modelled as an Additive White Gaussian Noise (AWGN) and the impulsive noise is based on the Middleton Class A noise model [4]. The Bit Error Rate (BER) performance of a Wireless Local Area Network (WLAN) channel in presence of Middleton Class A noise is investigated in [5]. The results suggest that impulsive noise has more effect in comparison with AWGN and degrades the BER performance of such a system more. In [6] the performance of using Orthogonal Frequency Division Multiplexing (OFDM) modulation for a PLC channel is analysed and compared with a single carrier modulation system and is found that the former enhances the BER performance over the later. The performance of PLC OFDM system in the presence of impulsive noise is simulated in [7]. It is observed that an Impulsive noise can degrade the performance of OFDM-based on PLC systems significantly. Therefore, channel coding is important to combat the impairments in this hostile medium, where the encoding and decoding of convolution codes over generated symbols for the transmission over impulsive noise channels. From the simulation results it can be observed that the excellent performance of the Viterbi decoder. The BER performance of bit-interleaved convolutionally coded OFDM under PLC channel characteristics and impulsive noise is investigated in [8]. The obtained simulation results show that, in the presence of impulsive noise, combination of convolutional coding and interleaving minimizes the effect of impulsive noise and improves the performance of PLC based on OFDM. In [9] an analytical approach is carried out to examine the performance of PLC channel in presence of fading and impulsive noise. The comparison results show that there is significant improvement in using OFDM over Binary Phase Shift Keying (BPSK).

This paper quantifies the BER performance of PLC channel for 16-Quadrarture Amplitude Modulation (QAM) with OFDM and Hamming Code in presence of impulsive noise modelled as the Middleton Class A noise source. Simulations are under taken in Matlab 2012a. Section II gives a brief overview of the basic concept of data transmission over PLC. Noise in the PLC channel with the Middleton Class A impulsive noise is discussed in section III. In section IV simulations for the OFDM PLC are given in Matlab 2012a followed by the results. Conclusions are given in section $\mathrm{V}$.

\section{PLC DATA TRANSMISSION}

A PLC can be modelled as in Fig. 1. The model consists of a PLC transmitter, the PLC channel with noise and the receiver block. According to the model, the received signal $r(t)$ is given by [10]: 


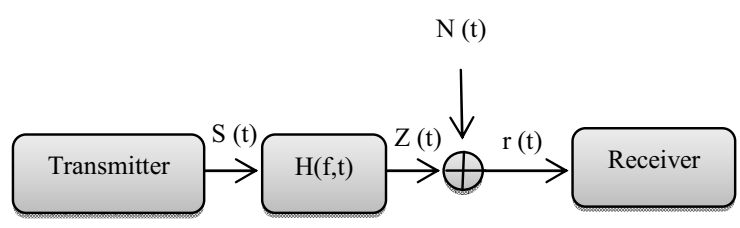

Fig.1. The basic PLC model.

$$
r(t)=s(t)^{*} h(t)+n(t)
$$

where:

$s(t)$ is the signal injected into the channel by the transmitter, $h(t)$ is the impulse response of the channel and $n(t)$ is the noise in the channel.

According to [11] there are various models available for PLC channel such as the Zimmermann and Dosteret model, Philipps model and the Anatory et al. model. The multipath model proposed by Philpps and Zimmermann is a widely used model for investigating the data transmission over power lines and is given by [11]:

$$
H(f)=\sum_{i=1}^{N} g_{i} e^{-\left(a_{0}+a_{1} f^{k}\right)^{d_{i}}} \cdot e^{-j 2 \pi f d_{i} / v_{p}}
$$

where:

$H(f)$ is the frequency response of the channel, $g_{i}$ is the weighting factor,$d_{i}$ the length of the data transmission path, $k$ is the exponent of the attenuation factor; $d i / v_{\mathrm{p}}$ stands for the time delay and $a_{0}$ and $a_{1}$ are attenuation parameters.

As in PLC frequency shift keying (FSK), amplitude shift keying (ASK) and phase shift keying (PSK) are used for low- data rate $(300 \mathrm{Kbps})$, for higher data rate transmission of more than $1 \mathrm{Mbps}$ modulations such as M-ary PSK, Mary QAM and OFDM are used [12]. In OFDM as the symbol duration increases for the lower rate subcarriers, the amount of dispersion in time caused by multipath delay reduces, thereby reducing the inter-symbol interference (ISI) leading to higher data rates.

\section{IMPULSIVE NOISE IN PLC}

Noise in PLC can be classified as a background and impulsive noise. Background noise is stationary and can be modelled by the Gaussian distribution [12]. An impulsive noise originates from variation sources such as heating processes, microwave ovens, electric motors and any other electrical sources. There are number of statistical models for characterization of an impulsive noise such as the Bernoulli-Gaussian and PoissonGaussian model [13]. An accurate model which is widely used is the Middleton Class $A$ based on the PoissonGaussian model. As it reported in [13] the probability of impulsive noise with $m$ impulsive noise events in a time interval $T$, for Poisson distribution parameter $\lambda$ can be modelled as:

$$
p=\frac{(\lambda T)^{m} e^{(-\lambda T)}}{m !}
$$

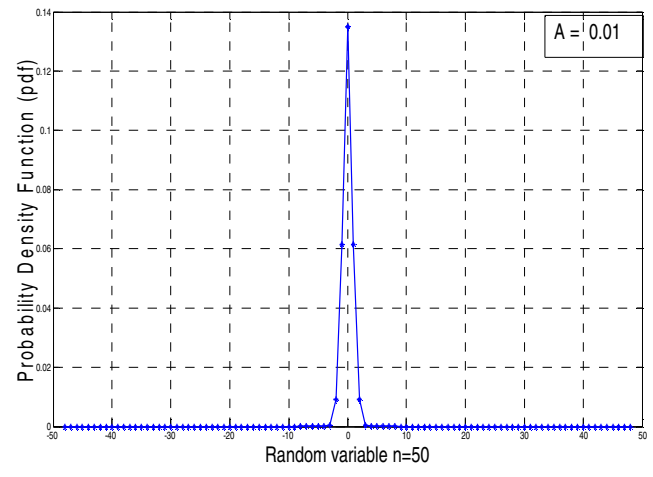

Fig.2. Pdf of the impulsive noise with impulsive index $A=0.01$.

If the impulsive index is given by $\mathrm{A}=\lambda \mathrm{T}$ then the Probability Density Function (pdf) of the Middleton Class A impulsive noise is given by [13]:

$$
p A(n)=\sum_{m=0}^{\infty} e^{-A} \frac{A^{m}}{m ! \pi N_{0}} \frac{A(1+\Gamma)}{m+A \Gamma} \exp \left[\frac{-A(1+\Gamma)}{(m+A \Gamma)} \frac{|n|^{2}}{N_{0}}\right]
$$

where:

$\sigma^{2}=$ the total noise power (both Gaussian and impulsive). $\Gamma=\sigma_{G}^{2} / \sigma_{I}^{2}$, is the mean power ratio of the Gaussian to impulsive noise. $N_{0}=2 \sigma^{2}$, is the one sided Power Spectral Density (psd) of the total white noise.

The main advantage of using Middleton Class A is that an equivalent noise source can be constructed and simulated from real world measured data. This enables the opportunity for quantifying noise characteristics based on real world measurements on various devices.

In Fig. 2 the pdf of the Middleton Class A noise is given with an impulsive index $A=0.01$.

\section{SIMULATION AND RESULTS}

Results of simulations undertaken in Matlab to quantify the BER performance of a PLC system using a higher modulation constellation of 16-QAM with Hamming code in presence of impulsive noise are given in this section. Fig.3. illustrates the diagram of the OFDM system used for simulation. A forward error correcting (FEC) code such as the Hamming code is used to enhance the data rate. An interleaving block is used to arrange the coded bits in a way that erroneous bits will be randomly distributed over many code-words rather than only a few code-words, subsequently the data bits are sent to the QAM modulator for mapping. Next is the OFDM stage which uses pilot signals to detect the channel response. The data stream is split into 64 parallel streams (by use of serial to parallel converter) that modulate 16 subcarriers using the Inverse Fast Fourier transform (IFFT). The Cyclic Prefix (CP) is inserted to reduce Inter-symbol interference (ISI). The multipath channel model has been used as given in (2). Background and impulsive noise are injected through the Channel. 


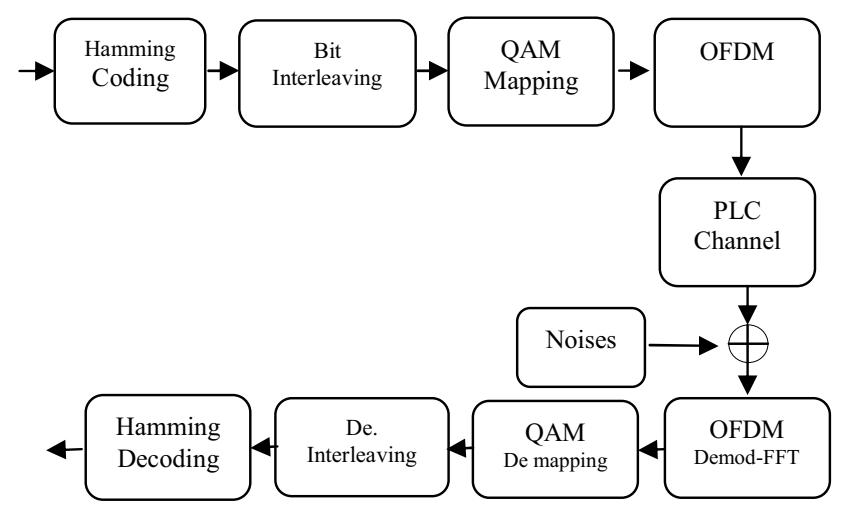

Fig.3. Diagram of an OFDM system consisting of a transmitter, a PLC channel and a receiver.

The effect of background and impulsive noise on the signal amplitude are given in Fig.4. As observed the impulsive noise distorts the signal amplitude while propagating through the channel. For 16 QAM the effect of variation in the impulsive index $A$ from 0.001 to 10 on the BER is shown in Fig.5. A high value of $A$ corresponds to low amplitude pulses with frequent occurances, while a low value of $A$ corresponds to high amplitude and less frequent pulses. As $A$ increases the BER performance improves with increasing SNR. A low value of $A$ would correspond to a signifant fault interference in the

power line likely as a result of a major fault, while higher values of $A$ would be the more regular low power interference caused due to common sources such as heating and electric motors.

Fig.6 shows the BER perforamance of the PLC through a multipath channel with background and impulsive noise.The BER performance is for $A=10$. The BER perfromance of the impulsive noise is worse than the background noise, even though for higher $A$ values. The BER peformance can be improved by using suitable filters. However the improvement is likely to occur for high

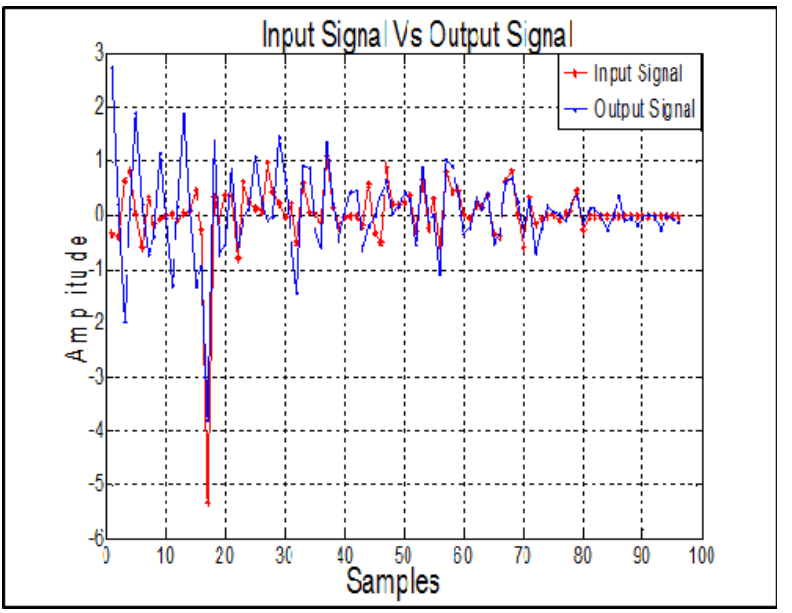

Fig.4.The Input signal Vs output affected by AWGN and Impulsive.

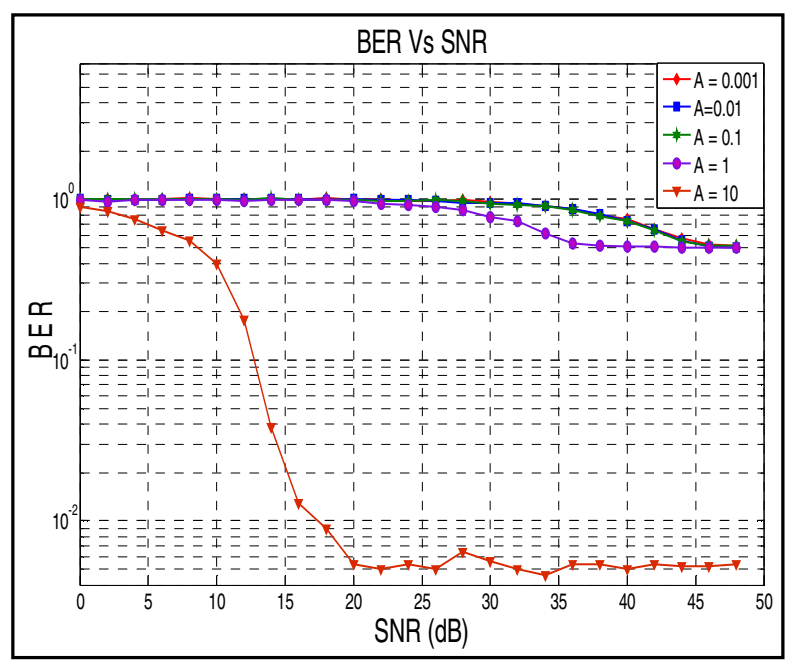

Fig.5. SNR Vs BER for different value of index (A) of Middleton Class A for the PLC channel.

values of $A$, for low $A$ values the improvement is not likely to be the same due to a much higher level of interference.

\section{CONCLUSIONS}

Main sources of noise in a power line are caused by the interference due to electric appliances connected to it which affect the data transfer over PLC channels. The main sources being the background and impulsive noise, are modelled and BER performance investigated for 16QAM constellation using OFDM technique. It is observed that BER Applying methods to mitigate and reduce the effect of impulsive noise over PLC systems for higher constellations to increase the data rates could be by means of alternate forward error correction/ convolution codes and suitable filters which are currently being investigated and would be published in a future publication.

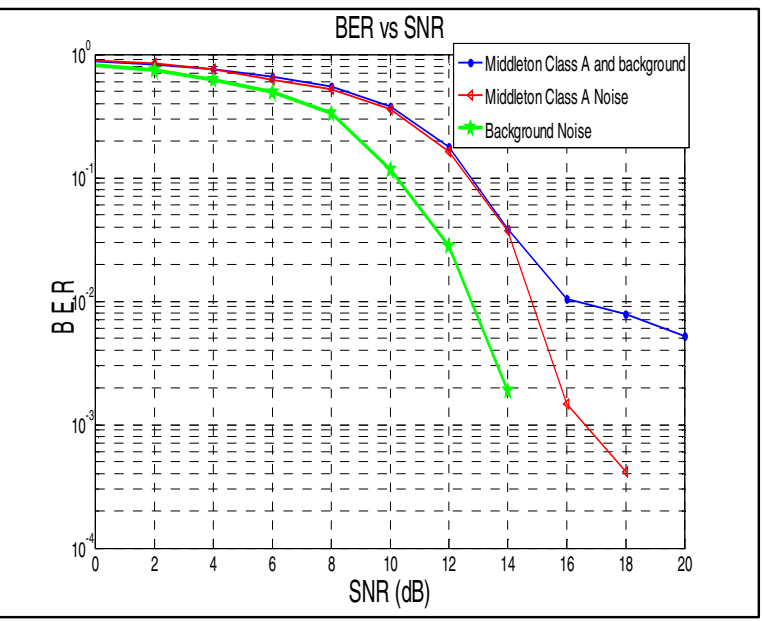

Fig.6. BER Vs SNR for multipath PLC channel. 


\section{REFERENCES}

[1] P. Sutterlin and W. Downey "A Power line communication tutorial challenges and technologies", Proc. IEEE $7^{\text {th }}$ Int. Symp. on Power Line Comms. \& its Appl., ISPLC 1998.

[2] A.Hosseinpournajarkolaei.H.Jahankhani.A.hosseinan-Far "Vulnerability Conciderations for Power Line Communication's (PLC) Supervisory Control and Data Acquisition". Int.J. Electronic Security and Digital Forensics, Vol.6. No2.pp.104-114.July 2014.

[3] P. Mlynek, M. Koutny and J. Misurec. "OFDM model for power line communication”. Proc. $4^{\text {th }}$ Int. Conf. on Comm. \& Info. Tech., CIT10, Greece, Jul 2010.

[4] M. M. Rahman And S. P. Majumder. "Performance Analysis of a Power Line Communication System Over a Non-white Additive Gaussian Noise Channel and Performance Improvement Using Diversity Reception". Proc. IEEE Industrial Electronics and Applications. Conf. on Network and Communication Technologies. ISIEA. Vol. 1.No.1. 2012.pp.304307.

[5] S. A. Bhatti. Q. Shan. I. A. Glover. R. Atkinson. And P.J. Moore "Impulsive Noise Modelling and Prediction of its Impact on the Performance of WLAN Receiver". Proc. $17^{\text {th }}$. European Signal Processing, EUSIPCO.Glasgow, Aug. 2009.

[6] L. Wang. G. Deconinck and E. Van Lil. "Performance analysis of Broadband Power line Communications under impulsive noise and crosstalk." Proc.URSI-Benelux. Conf. on Telecommuication and Microwaves.EAST-TELEMIC. Brussels.Sep. 2012.pp 1-3.

[7] C. Sekhararao. s.s.m.Reddy and K. Ravikumar . "Performance of coded OFDM in Impulsive Noise Environment".Proc. $15^{\text {th }}$. International Journal of Systems and Technologies. IJST. Volume 5, Issue 1. ISSN $0974-2107$.

[8] K.S. Al Mawali. Z.M. Hussain. "Performance of Bit-Interleaved Coded OFDM in Power Line Communication with Impulsive Noise".IEEE Int.Conf. Advanced Technologies for communication.Hai Phong.2009.

[9] M.M.Rahman. S.P Majumder." Analysis of Power Line Communication System Over a Non-white Additive Gaussian Noise Channel and Performance Improvement Using Diversity Reception". International Journal on Network and Communication technologies.Vol.1. No.1. June 2012.

[10] L. Selander. "Power Line Commucation:Channel Properties." department of Information Technology. Ronneby, Sweden.1999.

Available on:

http://www.eneresearch.com/knowledgebase/publications/thesises/ powerlinecom.pdf

[11] M. Zimmermann and K. Dostert, "A multipath model for the powerline channel," IEEE Trans. on Comms. vol., 50, no. 4, April 2002, pp. 553-559.

[12] T. Q. Bui. " Codded Modulation Techniques With Bit Interleaving and Iterative Processing for Impulsive Noise Channels". Degree of Master of Science. University of Saskatchewan, Saskatchewan, Canada. 2006.

Available on: http://homepage.usask.ca/ trb610/Mythesis2.pdf

[13] K.S. Al Mawali. "Techniques for Broadband Power Line Communications: Impulsive Noise Mitigation and Adaptive Modulation." Degree of Doctor of Philosophy. School of Electrical and Computer Engineering. RMIT University. July 2011.

Available on:

http://researchbank.rmit.edu.au/eserv/rmit:13700/Al_Mawali.pdf 